\title{
Micro-Raman and photoluminescence studies of neutron-irradiated gallium nitride epilayers
}

\author{
R. X. Wang, S. J. Xu, S. Fung, ${ }^{\text {a) }}$ C. D. Beling, K. Wang, S. Li, Z. F. Wei, T. J. Zhou, \\ J. D. Zhang, and Ying Huang \\ Department of Physics, The University of Hong Kong, Pokfulam Road, Hong Kong, \\ People's Republic of China \\ M. Gong \\ Department of Physics, Sichuan University, Chengdu, People's Republic of China
}

(Received 6 December 2004; accepted 7 June 2005; published online 12 July 2005)

\begin{abstract}
GaN epilayers grown on sapphire substrate were irradiated with various dosages of neutrons and were characterized using Micro-Raman and photoluminescence. It was found that the $\mathrm{A}_{1}(\mathrm{LO})$ peak in the Raman spectra clearly shifted with neutron irradiation dosage. Careful curve fitting of the Raman data was carried out to obtain the carrier concentration which was found to vary with the neutron irradiation dosage. The variation of the full width at half maximum height of the photoluminescence was consistent with the Raman results. The neutron irradiation-induced structural defects (likely to be $\mathrm{Ge}_{\mathrm{Ga}}$ ) give rise to carrier trap centers which are responsible for the observed reduction in carrier concentration of the irradiated GaN. (C) 2005 American Institute of Physics. [DOI: 10.1063/1.1999011]
\end{abstract}

In recent years, GaN and related compounds have attracted a great deal of interest ${ }^{1-3}$ due to their wide applications including blue/ultraviolet light-emitting devices, ${ }^{4,5}$ sun blind detectors, ${ }^{6}$ and high-power/high-temperature electronics. Although GaN-based light-emitting devices including laser diodes have been commercialized, the effects of various defects on the performance of the devices are still far from being fully understood. Further applications of GaN in optoelectronic devices demand a better understanding of the way optoelectronic properties of $\mathrm{GaN}$ films depend on some types of defects. It is well known that irradiation of high-energy particles, such as neutrons and electrons, can induce structural defects in semiconductors. In the past few decades, there were many reports discussing the irradiation-induced defects in silicon, ${ }^{7} \mathrm{GaAs},{ }^{8-10} \mathrm{GaP},{ }^{11}$ and $\mathrm{InP}^{12}{ }^{12}$ However, only a few studies ${ }^{13,14}$ have been reported on the effects of neutron irradiation on the microstructural and optical properties of $\mathrm{GaN}$ thin films. In this letter, we report on neutron irradiation-induced defects and their effects on the carrier concentration of GaN epilayers using Raman scattering and photoluminescence (PL) techniques. It is found that, in comparison with the as-grown material, the neutron-irradiated samples exhibit a clear variation in the position and line shape of the $A_{1}(\mathrm{LO})$-mode Raman peak as well as in the full width at half maximum (FWHM) height of the PL spectra as the neutron irradiation dosage changes. Careful curve fitting for the Raman peak, together with theoretical modeling based on the plasmon frequency, is used to determine the carrier concentrations of the neutron-irradiated GaN. The reduction of carrier concentration observed in the irradiated material is explained in terms of structural damage/or defects induced by neutron irradiation acting as carrier trapping centers.

The GaN wafer used in the study was grown on a sapphire (0001) substrate using the hydride vapor phase epitaxy

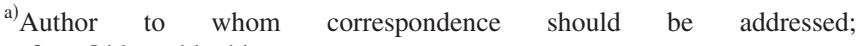
sfung@hkucc.hku.hk
}

method. Using Hall measurements, the carrier concentration and mobility of the GaN were found to be $3.3 \times 10^{17} \mathrm{~cm}^{-3}$ and $258 \mathrm{~cm}^{2} \mathrm{~V}^{-1} \mathrm{~s}^{-1}$, respectively. To study the effect of neutron radiation on $\mathrm{GaN}$, the wafer was cut into small pieces with an area of $4 \mathrm{~mm} \times 4 \mathrm{~mm}$. The samples were irradiated with neutrons (with a fast to thermal neutron ratio of $30 \%$ / $70 \%$ ) under different dosages, i.e., $1 \times 10^{15}, 5 \times 10^{15}$, and 1 $\times 10^{16} \mathrm{~cm}^{-2}$. In the following, these three samples are referred to as "low", "middle" and "high" dosage, respectively with the as-grown sample being used as a reference. The irradiated samples were not annealed.

For the PL measurements, the samples were mounted on the cold finger of a Janis closed-cycle cryostat with a temperature range of $4 \mathrm{~K}-300 \mathrm{~K}$ and were excited by the $325 \mathrm{~nm}$ line of a Kimmon $\mathrm{He}-\mathrm{Cd}$ continuous-wave laser with an output power of $40 \mathrm{~mW}$. The PL setup has been described in detail elsewhere. ${ }^{15}$ The micro-Raman scattering experiments were carried out under a backscattering geometry with a combination of instruments, namely a monochromator equipped with a 1800 lines $/ \mathrm{mm}$ grating, a microscope, a charge coupled device detector cooled by liquid nitrogen and a notch filter. The $488 \mathrm{~nm}$ line of a Coherent $\mathrm{Ar}^{+}+\mathrm{Kr}^{+}$mixed gas laser was employed as the excitation light in the Raman measurements and the typical spatial resolution of the Raman instrument was about $1 \mu \mathrm{m}$. Again, the detailed description on the confocal micro-Raman system can be found in a previous publication. ${ }^{16}$ All Raman scattering experiments were performed at room temperature.

Figure 1 shows the measured micro-Raman spectra of the $\mathrm{GaN}$ irradiated by different dosages of neutrons. The intensity of $A_{1}(\mathrm{LO})$ peak in each curve is normalized to unity. For clarity, the curves are vertically shifted. From Fig. 1, it can be seen that the $A_{1}(\mathrm{LO})$ mode of the neutron-irradiated GaN samples shifts toward the low-frequency side. Moreover, when compared to the as-grown sample, the line shape and the FWHM of the $A_{1}(\mathrm{LO})$ mode of these samples also change with neutron dosage. By comparison, the $\mathrm{E}_{2}$ (high) mode of all the samples including the as-grown one does not 


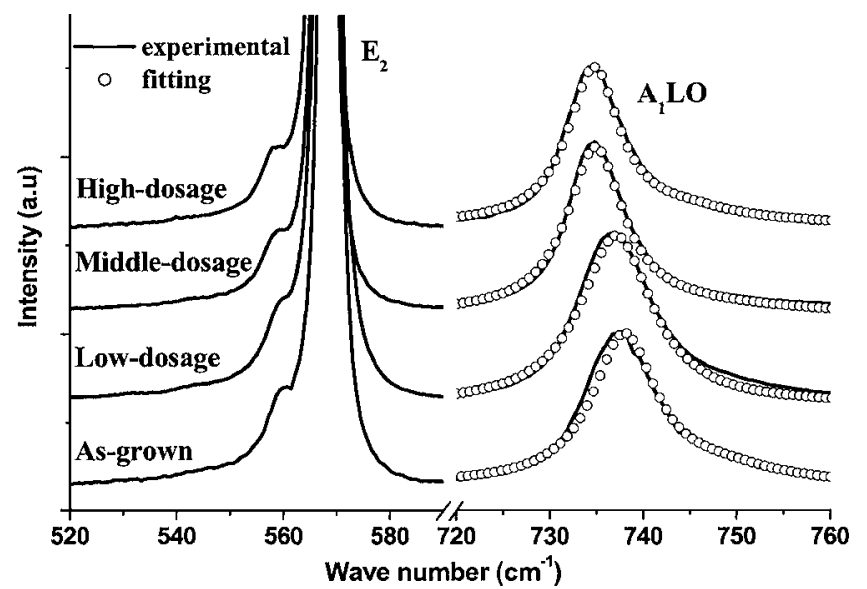

FIG. 1. Micro-Raman spectra of GaN samples, both as-grown and irradiated by neutron with different dosages.

change, confirming that the $\mathrm{A}_{1}(\mathrm{LO})$ mode's redshift seen in the irradiated samples is not due to any systematic experimental effect. It is known that the plasma mode of a free electron gas can couple with the longitudinal optical (LO) phonon lattice vibration mode in semiconductors giving rise to the so-called coupled mode whose frequency is closely related to the electron density. ${ }^{1,3,17,18}$ In order to estimate the free electron density in the neutron-irradiated GaN samples, the $\mathrm{A}_{1}(\mathrm{LO})$ modes peak shape was fitted to the functional form: ${ }^{17-20}$

$$
I(\omega)=K A(\omega) \operatorname{Im}\left(-\frac{1}{\epsilon_{\infty}\left[1+\frac{\omega_{L}^{2}-\omega_{T}^{2}}{\omega_{T}^{2}-\omega^{2}-i \omega \Gamma}-\frac{\omega_{p}^{2}}{\omega(\omega+i \gamma)}\right]}\right),
$$

where

$$
\begin{aligned}
A(\omega)= & 1+2 C \frac{\omega_{T}^{2}}{\Delta}\left[\omega_{p}^{2} \gamma\left(\omega_{T}^{2}-\omega^{2}\right)-\omega^{2} \Gamma\left(\omega^{2}+\gamma^{2}-\omega_{p}^{2}\right)\right] \\
& +C^{2}\left(\frac{\omega_{T}^{4}}{\Delta\left(\omega_{L}^{2}-\omega_{T}^{2}\right)}\right)\left\{\omega _ { p } ^ { 2 } \left[\gamma\left(\omega_{L}^{2}-\omega_{T}^{2}\right)\right.\right. \\
& \left.\left.+\Gamma\left(\omega_{p}^{2}-2 \omega^{2}\right)\right]+\omega^{2} \Gamma\left(\omega_{p}^{2}+\gamma^{2}\right)\right\}
\end{aligned}
$$

and where $\Delta$ and the plasma frequency $\omega_{P}$ are given by

$$
\begin{aligned}
& \left.\Delta=\omega_{p}^{2} \chi\left(\omega_{T}^{2}-\omega^{2}\right)^{2}+(\omega \Gamma)^{2}\right\rfloor+\omega^{2} \Gamma\left(\omega_{L}^{2}-\omega_{T}^{2}\right)\left(\omega^{2}+\gamma^{2}\right), \\
& \omega_{P}=\sqrt{\frac{4 \pi n e^{2}}{m^{*} \varepsilon \varepsilon_{0}}},
\end{aligned}
$$

where $\omega_{L}$ and $\omega_{T}$ are, respectively, the frequencies of the LO and transverse optical phonons, respectively. $K, n, \gamma$, and $\Gamma$ are, respectively, the constant coefficient, the carrier density, the plasmon damping constant, and the phonon damping constant. Here, we use $\omega_{T}=533 \mathrm{~cm}^{-1}, \omega_{L}=735 \mathrm{~cm}^{-1}, \varepsilon$ $=5.35^{1}, m^{*}=0.2 m_{0}^{2}$, and $\mathrm{C}=0.4$ (Ref. 19) for GaN. The fitting parameters are listed in Table I and the carrier concentrations of the samples derived are thus $2.5 \times 10^{17} \mathrm{~cm}^{-3}, 1.3$ $\times 10^{17} \mathrm{~cm}^{-3}, 1.2 \times 10^{17} \mathrm{~cm}^{-3}$, and $2.9 \times 10^{17} \mathrm{~cm}^{-3}$ for the low dosage, middle dosage, high dosage, and as-grown

\begin{tabular}{|c|c|c|c|c|c|}
\hline \multicolumn{2}{|c|}{ Irradiation dosage } & \multirow{2}{*}{$\frac{\text { As-grown }}{120}$} & \multirow{2}{*}{$\frac{1 \times 10^{15}}{105}$} & \multirow{2}{*}{$\frac{5 \times 10^{15}}{55}$} & \multirow{2}{*}{$\frac{1 \times 10^{16}}{50}$} \\
\hline & $\omega_{P}$ & & & & \\
\hline Fitting & $\gamma$ & 300 & 265 & 259 & 218 \\
\hline parameters & $\Gamma$ & 5.4 & 7.1 & 7.0 & 6.8 \\
\hline Derived & $\begin{array}{l}\text { Carrier concentration } \\
\quad\left(\times 10^{17} \mathrm{~cm}^{-3}\right)\end{array}$ & 2.9 & 2.5 & 1.3 & 1.2 \\
\hline PL & FHM (meV) & 3.34 & 3.55 & 3.66 & 3.84 \\
\hline
\end{tabular}
samples, respectively. Since GaN is transparent to the 488 $\mathrm{nm}$ excitation light used in the Raman measurements, the Downloaded 20 Apr 2007 to 147.8.143.135. Redistribution subject
TABLE I. Values of the fitting parameter for Raman $A_{1}(L O)$ mode and the PL FWHM.

Raman signal from the sapphire substrate is thus observed. Therefore, the substrate phonon peak $\left(\sim 749 \mathrm{~cm}^{-1}\right)$ is subtracted in order to obtain a clean baseline. ${ }^{19}$

Usually, carrier removal is mainly due to the trapping of majority carriers by structural defects. ${ }^{8,9,21,22}$ To test this idea and to investigate the crystal structure of the irradiated GaN, PL spectra of the samples were taken. Figure 2 shows the low-temperature PL spectra of the samples. Unlike the cases studied by Kuriyama et al., ${ }^{13}$ for the present study, the bandedge transition at $\sim 3.48 \mathrm{eV}$ still dominates even for the sample irradiated with the highest neutron dosage since the neutron dosages employed by us are lower by at least three orders of magnitude. However, the FWHM, intensity, and even the position of the band-edge transition vary with irradiation dosage. The FWHM of the PL spectra is seen to increase with neutron dosage. Moreover except for the lowdosage sample, the PL intensity drops as the neutron dosage increases. The PL intensity of the low-dosage sample is slightly higher than that of the as-grown sample. However, this is not definitive as the effect is of the same order of magnitude as our systematic error. It is known that both the FWHM and peak intensity of the PL spectrum from a sample are closely related to its quality. ${ }^{23}$ For samples with the same crystal structure and chemical composition, the widened PL peak width certainly indicates a deterioration of crystalline quality. Thus, from the PL data, it can be concluded that, as the neutron dosage increases, the structural quality of the samples deteriorates. The physical origin of the blueshift of the band-edge transition as the neutron dosage increases is not clear at present. It is likely to be due to the reduction of the carrier concentration as the $\mathrm{E}_{2}$ (high) mode peaks in the

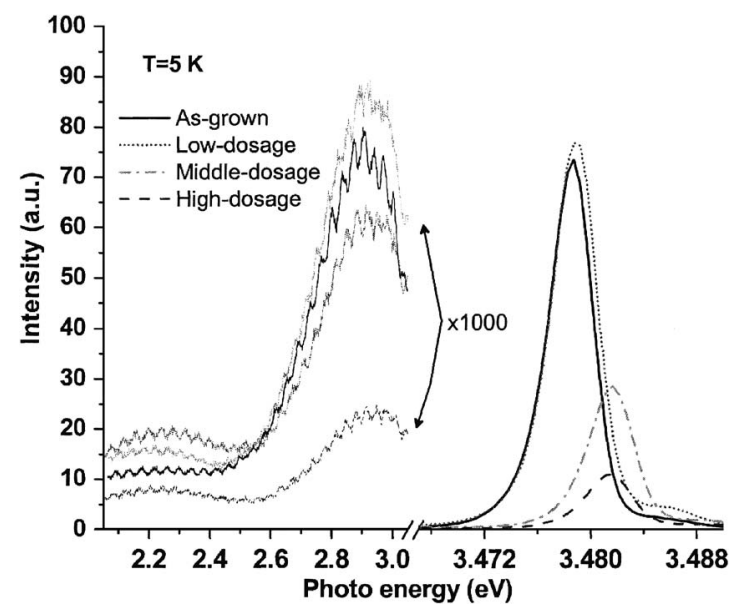

FIG. 2. Low-temperature PL spectra of GaN samples, both as-grown and

irradiated by neutron with various dosages. 
Raman spectra shown in Fig. 1 do not shift. The $\mathrm{E}_{2}$ (high) mode is very sensitive to the strain of the GaN epilayer. ${ }^{16}$

Now we turn to discuss the defect-related emission peaks. During the irradiation process, the GaN structure is not only bombarded by thermal neutrons that transmute ${ }^{69} \mathrm{Ga},{ }^{71} \mathrm{Ga},{ }^{72} \mathrm{Ga}$ to ${ }^{70} \mathrm{Ge},{ }^{72} \mathrm{Ge}$ and $/$ or ${ }^{14} \mathrm{~N},{ }^{15} \mathrm{~N}$ to ${ }^{16} \mathrm{O}$ (Ref. 13) but also by fast neutrons producing point defects. Both of these processes cause the crystal structure to slightly distort with a subsequent production of strain. ${ }^{8,10,24}$ These defects can be shallow traps and/or deep level defects and even complex defect centers. ${ }^{13,14,24}$ A weak blue luminescence peak centered at $\sim 2.9 \mathrm{eV}$ can be observed for all the samples. However, its relative intensity with respect to the band-edge transition changes with neutron dosage. Except for the low-dosage case, the relative intensity of the $2.9 \mathrm{eV}$ broad peak increases as the neutron dosage increases. This indicates that the emission peak should be related to the neutron irradiation. Kuriyama et al. ${ }^{13}$ also observed a $2.9 \mathrm{eV}$ emission in their neutron-transmutation-doped $\mathrm{GaN}$ which was annealed at $1000^{\circ} \mathrm{C}$ for $60 \mathrm{~min}$ and attributed it to the neutron transmuted $\mathrm{Ge}$ atoms at $\mathrm{Ga}$ sites. One theoretical study shows that the $\mathrm{Ge}_{\mathrm{Ga}}$ can produce a $D X$-like state through capturing an electron. ${ }^{24}$ Furthermore, this negatively charged $\mathrm{Ge}_{\mathrm{Ga}}$ state can act as a metastable center and introduces a singlet state which almost degenerates with the valence-band top and a second singlet state $0.5 \mathrm{eV}$ below the bottom of the conduction band. ${ }^{24,13}$ Therefore, the neutron transmuted $\mathrm{Ge}$ should be responsible for the relative increase of the $2.9 \mathrm{eV}$ peak intensity. The blue emission from the metastable states in $\mathrm{GaN}$ epilayers has been observed by $\mathrm{Xu}$ et $a l^{25}$ They also found that the blue emission band was closely related to the yellow emission. Note that the yellow emission of the samples in the present study is much weaker and thus from which reliable information cannot be derived.

In conclusion, $\mathrm{GaN}$ samples irradiated by different neutron dosages have been investigated using Raman scattering and PL spectroscopic techniques. It has been observed that the $A_{1}(\mathrm{LO})$ peak position in the Raman scattering spectra clearly shifts toward the lower-frequency side and the FWHM of PL spectra becomes larger with increasing neutron irradiation dosage. From careful analysis of experimental Raman spectra, it is found that the carrier concentration of GaN samples decreases as the neutron irradiation dosage increases. This has been explained on the basis of the $D X$-like centers induced by neutron irradiation acting as the carrier trapping centers thereby compensating for the carrier concentration of the GaN samples.
The work described in this letter is partially supported by the grants from the Research Grant Council of the Hong Kong Special Administrative Region, China (under Project Nos. HKU7036/03P, HKU7021/04P, HKU7004/03P, and HKU1/00C). One of the authors (M. G.) also acknowledges support from the National Nature Science of China (Grant No. 60076010) and a grant from the National Key Lab of Silicon Materials of China.

${ }^{1}$ H. Harima, H. Sakashita, T. Inoue, and S. Nakashima, J. Cryst. Growth 189, 672 (1998).

${ }^{2}$ N. Wieser, M. Klose, R. Dassow, F. Scholz, and J. Off, J. Cryst. Growth 189, 661 (1998).

${ }^{3}$ D. Meister, M. Böhm, M. Topf, W. Kriegseis, W. Burkhardt, I. Dirntorfer, S. Rösel, B. Frangis, B. K. Myer, A. Hoffmann, H. Siegle, C. Thomsen, J. Christen, and F. Bertram, J. Appl. Phys. 88, 1811 (2000).

${ }^{4}$ J. K. Sheu, Y. K. Su, G. C. Chi, M. J. Jou, and C. M. Chang, Appl. Phys. Lett. 72, 3317 (1998)

${ }^{5}$ F. D. Aurent, S. D. Goodman, F. K. Koschnick, J. M. Spaeth, B. Beaumont, and P. Gibart, Appl. Phys. Lett. 74, 2173 (1999).

${ }^{6}$ Y. K. Su, F. S. Juang, and M.-H. Chen, Jpn. J. Appl. Phys., Part 1 42, 2257 (2003).

${ }^{7}$ M. Coeck, N. Balcaen, T. Van Hoecke, B. Van Waeyenberge, D. Segers, C. Dauwe, and C. Laermana, J. Appl. Phys. 87, 3674 (2000).

${ }^{8}$ J. G. Williams, J. U. Patel, A. M. Ougouag, and S. Y. Yang, J. Appl. Phys. 70, 4931 (1991).

${ }^{9}$ G. E. Brehm and G. L. Pearson, J. Appl. Phys. 43, 568 (1972).

${ }^{10}$ M. Satoh, K. Kuriyama, and T. Kawakubo, J. Appl. Phys. 67, 3542 (1990).

${ }^{11}$ M. Palczewska, J. Jasinski, K. Korona, M. Kaminska, E. D. Bourrent, and A. G. Elliot, J. Appl. Phys. 78, 3680 (1995).

${ }^{12}$ B. Mari, M. A. Hernandez-Fenollosa, and F. J. Navarro, J. Appl. Phys. 89, 7772 (2001).

${ }^{13}$ K. Kuriyama, T. Tokumasu, J. Takahashi, H. Kondo, and M. Okada, Appl. Phys. Lett. 80, 3328 (2002).

${ }^{14}$ C. W. Wang, J. Vac. Sci. Technol. B 20, 1821 (2002).

${ }^{15}$ S. J. Xu, H. J. Wang, S. H. Cheung, Q. Li, X. Q. Dai, M. H. Xie, and S. Y. Tong, Appl. Phys. Lett. 83, 3477 (2003).

${ }^{16}$ D. G. Zhao, S. J. Xu, M. H. Xie, S. Y. Tong, and H. Yang, Appl. Phys. Lett. 83, 677 (2003).

${ }^{17}$ W. Gotz, N. M. Johnson, H. Amano, and I. Akasaki, Appl. Phys. Lett. 65, 463 (1994).

${ }^{18}$ H. Harima, S. Nakashima, and T. Uemura, J. Appl. Phys. 78, 1996 (1995).

${ }^{19}$ T. Kozawa, T. Kachi, H. Kano, Y. Taga, M. Hashimoto, N. Koide, and K. Manabe, J. Appl. Phys. 75, 1098 (1994).

${ }^{20}$ H. Harima, J. Phys.: Condens. Matter 14, R967 (2002).

${ }^{21}$ M. Senthil, R. Kesavamoorthy, and P. Magudapathy, Nucl. Instrum. Methods Phys. Res. B 179, 193 (2001).

${ }^{22}$ A. Castaldini, A. Cavallini, and L. Polenta, J. Phys.: Condens. Matter 12, 10161 (2000).

${ }^{23}$ J. Y. Shi, L. P. Yu, Y. Z. Wang, G. Y. Zhang, and H. Zhang, Appl. Phys. Lett. 80, 2293 (2002).

${ }^{24}$ P. Boguslawski and J. Bernholc, Phys. Rev. B 56, 9496 (1997).

${ }^{25}$ S. J. Xu, G. Li, S. J. Chua, X. C. Wang, and W. Wang, Appl. Phys. Lett. 72, 2451 (1998). 